\title{
Design of Integrated Routing System for Mobile Ad Hoc Networks Overlaying Peer-to-Peer Applications
}

\author{
Yan Annie Ding and David Everitt \\ School of Information Technologies, \\ University of Sydney, NSW 2006, Australia \\ \{annie, deveritt\}@it.usyd.edu.au
}

\begin{abstract}
This paper proposes a novel distributed routing system for integration between peer-to-peer (P2P) applications and mobile ad hoc networks (MANETs). This system takes advantage of the "zone" concept to reduce the multicast flooding. Significantly, the system investigates a mechanism to integrate key lookup in the application layer with routing in the link layer. The routing method provides a general-purpose technique that is not limited to any specific P2P applications. This paper presents the design of the routing system and sketches the layered architecture built according to the system functions.
\end{abstract}

\section{Introduction}

Peer-to-peer (P2P) overlay networks are self-organizing virtual networks over the IP-layer normally with TCP/IP connections. P2P services contribute to effective file sharing and resource locating in a computing environment without a central server. Mobile ad hoc networks (MANETs) are defined as wireless multi-hop networks formed dynamically and self-organized by mobile services for some purpose. Both of the networks have common characteristics in dynamicity, autonomy and "multi-hop" routing. These similarities motivate us to exploit the synergy [1] between P2P networks and MANETs.

P2P networks need high bandwidth network transmission and fast calculation speed, which can be fulfilled by the wired Internet. There are two main searching algorithms based on flooding [2] or Distributed Hash Table (DHT) [3], 4], [5], [6] in wired networks. These advanced P2P techniques are largely restricted in MANETs due to the autonomous distribution, scarce bandwidth and changing topology in physical links. The broadcast or flooding methods applied in the application layer are unable to supply smooth P2P applications under a MANET environment. The DHT based algorithm may induce excessive load. The severe situation is that the evoked broadcast storms [7] may break the whole system down when these wired methods are directly applied to MANETs.

With the growing demand in wireless Internet services and P2P applications such as instant messaging, ubiquitous computing and resource sharing, effective 
$\mathrm{P} 2 \mathrm{P}$ searching is very important, considering especially the vulnerable characteristics of MANETs. Although P2P systems and MANETs have many similar characteristics, they are completely different networks based on different layers and produce different broadcasts. When applying similar self-organizing $\mathrm{P} 2 \mathrm{P}$ applications to MANETs, the major problem is how to efficiently query and find the matching documents in the MANET environment with limited power, scarce bandwidth and changing topology. We consider the characteristics of MANETs and propose a novel realistic system - Multicast Key-based Zone Routing (MKZR) based on practical multi-hop flooding. We aim to build P2P applications over MANETs smoothly and exploit the synergy between them.

In this paper, we sketch the system architecture and present the design model for the MKZR system. Section 2 analyses the current problems and the corresponding MKZR system idea. Section 3 describes our system architecture and algorithms in detail. Section 4 compares some related work. Section 5 gives some conclusions to our system designs and future work.

\section{Current Problems and MKZR System}

When applying P2P applications over "one-hop" MANETs with effective searching in one hop region, the method of flooding and caching contents 8$]$ is a practical method. When overlaying P2P applications to larger MANETs, we can address some critical problems and the design notion for the MKZR system as follows:

\subsection{Broadcast Storm}

The broadcast storm problem in MANET was discussed in [7]. Here we refer to the broadcast storm problem from pure broadcast or flooding mechanism in $\mathrm{P} 2 \mathrm{P}$ mobile ad hoc routing systems.

Pure P2P flooding is fragile due to the congestion after frequent query broadcasts. These query broadcasts are virtual broadcasts based on the application layer. Furthermore they can induce real broadcasts in the network layer. However the growing demands of $\mathrm{P} 2 \mathrm{P}$ applications in MANETs aggregate a lot of text processing and querying, which unavoidably burden the network load in MANETs. The overhead in this network architecture is as high as $\mathrm{O}\left(N^{2}\right)[9$. This is disastrous in resource-scarce networks like MANETs. We reviewed current related works and found that systemic $\mathrm{P} 2 \mathrm{P}$ routing algorithm design is still in its early stage in wireless MANETs. We argue that effective P2P routing mechanism is an important promising research area presenting the base for modern information sharing and ubiquitous computing under the P2P MANET environment.

In order to reduce the broadcasts, we use two novel and effective methods. Firstly, our MKZR routing system aims to integrate P2P searching and link layer routing. Secondly the MKZR introduces the "zone" concept to quickly locate the destination node. 


\subsection{Redundant Caching}

Current caching of pairs $<$ key, value $>$ information in each node can reduce the querying time and hence result in effective use of network bandwidth. Caching systems in P2P MANETs obtain information mainly through dissemination and as a result each node caches the same contents of pairs. This method needs to be improved to save resources and reduce the administrative overhead.

The MKZR is motivated to be a routing system, which caches routing information instead of application layer keyword matching information $<$ key, value $>$. Each node caches different routing information instead of the same content of keyword matches. In addition, our system will attempt to cache the routing information matches of $<$ key, node address $>$. The node address belongs to the name space of link layer identification.

In the repository, an implementation of data index table presents the local data information indices.

\section{Models and Algorithms}

We propose the Multicast Key-based Zone Routing (MKZR) system based on the following main ideas:

"Zone" Concept. The network is divided into several zones where the hop distance between the central node and other nodes in the same zone is restricted to be within a specified radius, similar to Zone Routing Protocol (ZRP) [10. The radius distance is calculated in hops and the peripheral nodes are situated around the radius distance. If a key search needs to find a new route, it will first multicast within the zone. If that fails, it will take advantage of the peripheral nodes to keep searching within their own zones. After the simulation, we will attempt to implement more functions such as storing more sharing information in peripheral nodes to reduce the searching overhead. We will compare the performance of the added functions.

Routing Integration. Our system aims to form a "key-based" whole system routing, which the key searching of the self-organized peers in the application layer can combine with the link layer routing in MANETs. The whole network is an integrated coordinating system to finish a key search or key lookup task. The integrating of P2P key searching and link layer routing of MANETs operates as a "resolver" in the network layer to transfer the IP address to the node ID in the link layer via a one-to-one mapping. The routing information stored in the application layer will be changed to $<$ key, node ID route $>$ instead of $<$ key, IP address $>$ at the last stage of the implementation. At the first stage the $<$ key, IP address $>$ is still used for the whole system simulation.

Cache Content. The cache system collects routing information as its content. There are two different tables in each mobile node: one is a routing table related with key $<$ key, node ID $>$ and the other is a neighbour table related with our zone division. The neighbour table is designed to reduce the broadcast overhead incurred from searching. 


\subsection{Layered Architecture}

As $\mathrm{P} 2 \mathrm{P}$ technologies mature in wired networks, $\mathrm{P} 2 \mathrm{P}$ is considered as a decentralized symmetric computing model which various applications can run above. Our system aims to build a system model or protocol for a MANET running $\mathrm{P} 2 \mathrm{P}$ applications. The mobile nodes in the model are randomly moving with P2P networks overlaid. The lower layer behaviour is "Make a move" or "Have a rest", etc., as shown in Fig 3 . The system architecture is layered as Fig. 1 according to our requirement analysis. The system is virtually divided as various subsystems or sub-models based on their different executing functions, e.g., the Issuer can send query or reply messages. The functions of each subsystem will be described in Section 3.3 .

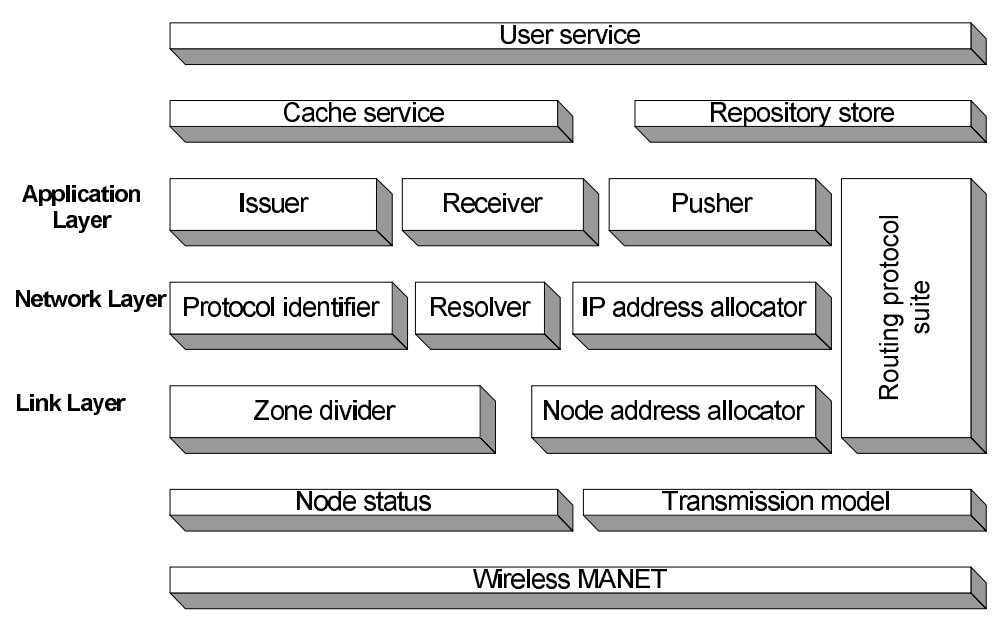

Fig. 1. Layered system architecture

\subsection{Message Format}

The system defines five types of message, $Q U E R Y, R E P L Y, Q U E R Y \_Z O N E$, REPLY_NULL and PUSH which are shown in Fig. 2 .

\subsection{System Function Overview}

A key search starts from the application layer model of "Issuer". The network layer model "Resolver" resolves the node ID in the link layer name space to an IP address. Protocol identifier can distinguish the different messages. Zone divider can divide zones according to the hop information in the protocol format. We list some main system functions in Fig. 3, We gradually describe our system model and functions from the application layer. 
QUERY:

\begin{tabular}{|l|l|l|l|l|l|l|}
\hline Type $=0$ & Key & SRC & SEQ & RTT & TTL & R \\
\hline
\end{tabular}

REPLY:

\begin{tabular}{|l|l|l|l|l|l|l|}
\hline Key & SRC & SEQ & RT & TTL & DES $>>$ NULL & R \\
\hline
\end{tabular}

\section{QUERY ZONE}

\begin{tabular}{|l|l|l|l|l|l|l|}
\hline Type $=1$ & Key & SRC & SEQ & RTT & TTL & R \\
\hline
\end{tabular}

\section{REPLY_NULL:}

\begin{tabular}{|l|l|l|l|l|l|l|}
\hline Key & SRC & SEQ & RTT & TTL & DES=NULL & R \\
\hline
\end{tabular}

\section{PUSH:}

\begin{tabular}{|l|l|l|l|}
\hline ID File & Index & DEX & R \\
\hline
\end{tabular}

Fig. 2. Message format

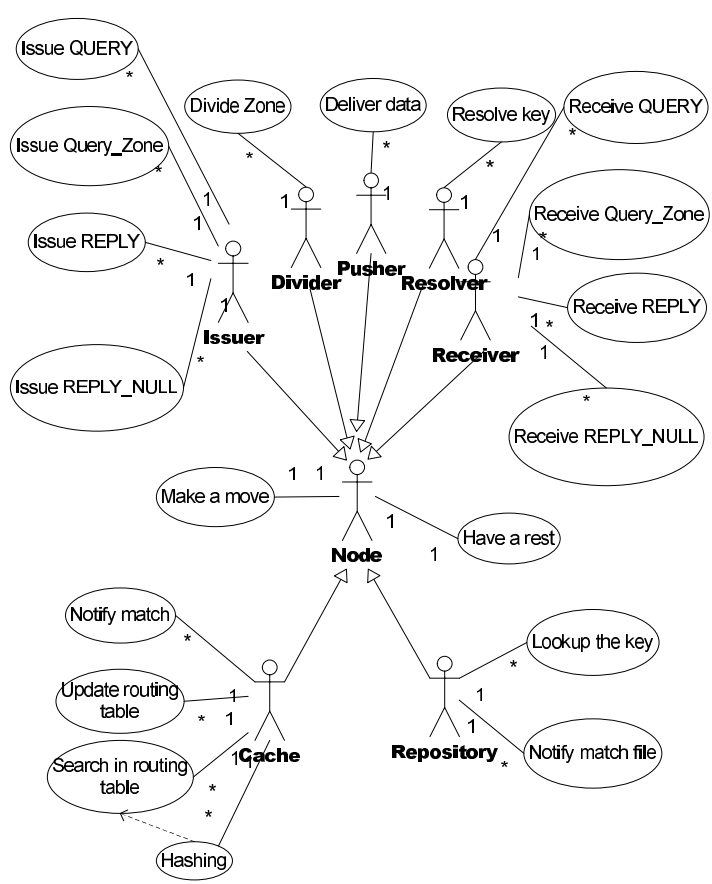

Fig. 3. System function overview

\subsection{Application Layer}

In our architecture model design, a routing task starts from a key search of a peer node in the application layer. 


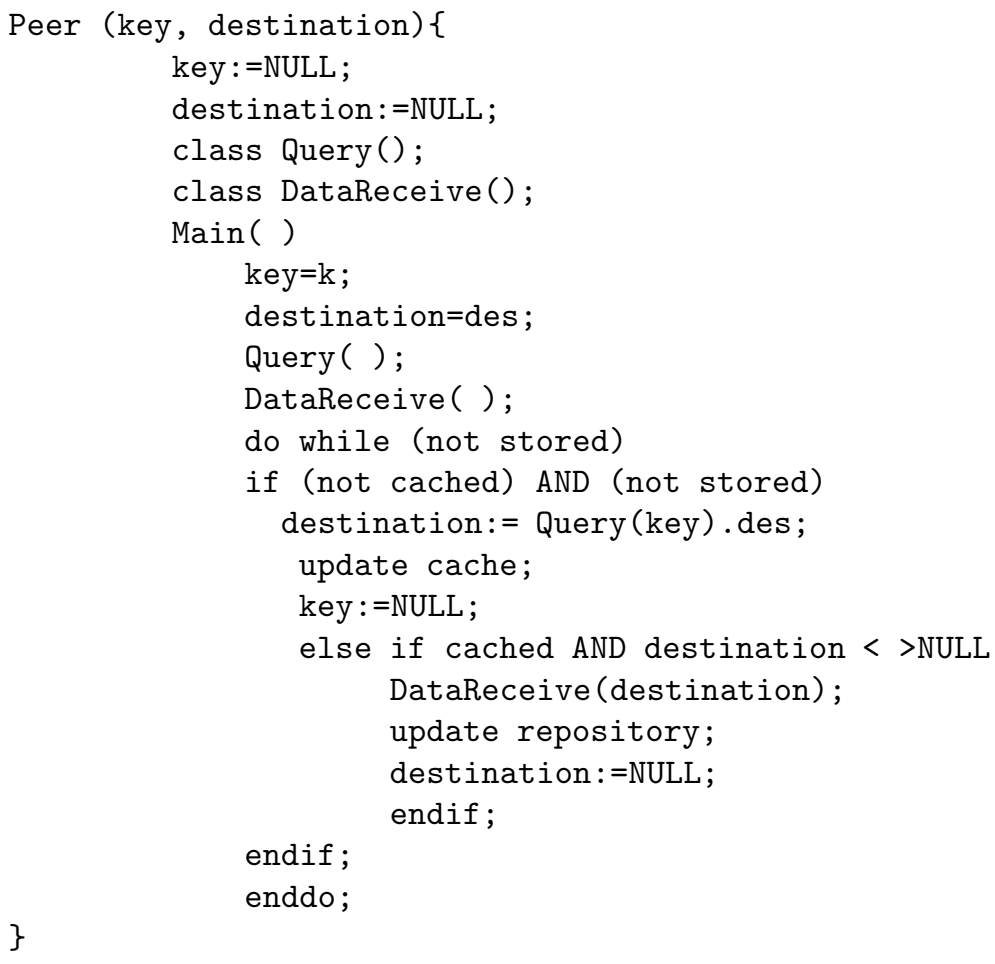

Query Algorithm. MKZR provides an efficient key-searching algorithm by combining querying in the application layer with routing in the network layer. When a peer node issues a query for matching a key, it firstly checks its local index key table in Repository. If there is no match, it will search in the routing table. If it finds the key match in cache, the node directly sends a data request to the destination node and then is pushed back the data needed. If it does not find the route, the $Q U E R Y$ message piggybacks the key on the base of a link-layer broadcast message, which is distributed by link-layer flooding. The checking order is "local index key table - routing table - other nodes". The $Q U E R Y$ message builds reverse paths to the issuer node in all the intermediate nodes it passed during the process to the destination node. In addition, when node multicasts a $Q U E R Y$ message there are two following situations need to be considered:

Destination Node Within the Zone Area. If there is a node that contains the content or owns the routing information of the key, this node will send a REPLY message containing the routing information such as the destination IP address ("DES" in message) of the key match directly to the issuer node. After the key-issuing node receives this $R E P L Y$, it will update its routing table and decide to stop the query. Those non-peripheral nodes without the key information are designed to simply discard the $Q U E R Y$ message. Those peripheral nodes without the key routing information will send a $R E P L Y$ message without any 
data included. We define this special REPLY message as REPLY_NULL in Section 3.2. In our implementation, only peripheral nodes of a zone can send this empty data message. The issuer node discards this message after receiving $R E P L Y \_N U L L$ if it obtains the matching information from other nodes.

Destination Node Outside the Zone Area. The query task will hand over to peripheral nodes if there is no match within the zone. These peripheral nodes will keep multicasting within the scope of their zone after the issuer node sends a $Q U E R Y \_Z O N E$ message. The notice must be sent with query key due to the last discarding operation.

Data Delivery. After the issuer node gets the routing information, it can build the direct connection to the destination node. The destination node can push the data to the issuer node after the issuer node request. The initial design ignores the information backup in $\mathrm{P} 2 \mathrm{P}$ applications. The system can replicate some information requested frequently in some bone node such as peripheral nodes.

\subsection{Network Layer and Link Layer}

There is a "Resolver" in the network layer to complete the resolution between an IP address and a link layer node address. The implementation is important to the integrated routing between P2P networks and MANETs.

The protocol identifier model can extract the hop information. The system can then divide zones and implement neighbour node tables according to the hop information. The current P2P query algorithms or protocols are developed in wired networks which is not suitable to the MANETs as analyzed in Section2, The MKZR link layer protocol is designed on the basis of multicast flooding but merged with the idea of Ad Hoc On-Demand Distance-Vector Protocol (AODV) [11] and our zone concept described above.

\subsection{Integrated Routing}

Protocol Integration. Firstly, the query algorithms in the application layer combine with link layer protocols. MKZR piggybacks the application layer key onto the message format and sends it through the link layer protocol to the next hop. This type of integrated routing like MKZR between P2P networks and MANETs operates a "key-based" routing style to drive the link layer protocol. Secondly, each message contains a field SRC (Source) for the unique identifier of the sender mobile node and a field SEQ for an increasing sequence number. The implementation of SRC and SEQ (Sequence) guarantees the forwarding flow and correct reverse path. The idea is borrowed from AODV.

Name Space Integration. In P2P routing, the general method to cope with the naming of key or related IP address is to hash them into one name space. The commonly used hash technique is consistent hashing Secure Hash Algorithm 
(SHA-1) 12. Our approach is to use pairs $<$ keyword, IP address $>$ as the routing table entries and then hash them separately as $<$ key, peer ID $>$. We build a oneto-one mapping scheme between peer ID and link layer node ID. Furthermore, we will investigate the possibility of using the link layer node ID to hash as the peer ID in $\mathrm{P} 2 \mathrm{P}$ routing tables.

As a result of the above integration, the virtual broadcast overlaid in the application layer can directly find the link layer route to reduce the algorithm complexity from $\mathrm{O}\left(N^{2}\right)$ to $\mathrm{O}(N)$. In P2P MANETs, a key lookup can get to the destination node through the virtual application layer broadcasts to search the key and the network layer broadcasts to route the search information. The double broadcasts make $\mathrm{O}\left(N^{2}\right)$ routing steps in the pure flooding method. The MKZR protocol is a key-based application-layer driven network-layer routing protocol (We define this key-driven protocol). We propose to skip the virtual overlaying. This means that the virtual broadcasts induced by key searching are incorporated with the network layer routing broadcasts. If there is a pure keybased flooding method which is a network layer protocol like MKZR, then the performance of the pure key-based network flooding is $\mathrm{O}(N)$.

\section{Related Work}

Wireless P2P research is often based on broadcast or flooding. For example, 7DS (Seven Degrees of Separation) 13] is implemented to exchange data for web-browsing among peers without connecting to the Internet. End users can advertise what they have, or query data objects related to the URL or host MAC addresses and get the information through broadcasts. 7DS is an approach not for discovery routing but for P2P application services.

Similar to 7DS, Passive Distributed Indexing (PDI) [8] and Optimized Routing Independent Overlay Network (ORION) [14] can provide file sharing locally by the implementation of a file routing table. Far more than that, PDI and ORION presents a P2P file sharing system based on MANET. Their generalpurpose design may present the foundation for the later practical research in P2P MANETs. In particular, ORION introduces AODV techniques into the peer searching. ORION uses multicast flooding and stores file routing table and response routing table in the cache system for file sharing. However, data dissemination methods in both systems make the cache system in each node store the consistent content at the end.

Proem 15] is a general-purpose mobile computing platform for $\mathrm{P} 2 \mathrm{P}$ applications and developments. It integrates $\mathrm{P} 2 \mathrm{P}$ protocol, database and development tools. Messages are encoded using the XML language to communicate between peers. Proem does not consider how lower levels in MANETs are connected or self-organized. However, Dynamic P2P Source Routing (DPSR) [1] considers this point.

DPSR exploits the synergy between routing in $\mathrm{P} 2 \mathrm{P}$ systems and that in MANETs, which designs a network layer routing protocol to integrate Pastry with DSR. However DPSR needs further investigation when considering the 
seamless connection between application layer routing of Pastry and link layer routing.

The MIN architecture [16] proposes an evolving architecture overlaid on top of the application layer to integrate P2P applications with MANETs. The work is in its early stages.

\section{$5 \quad$ Summary}

Our proposal suggests a routing protocol especially for applying P2P applications over MANETs. We argue that the performance improvement for P2P MANETs is dependent on the breakthrough of MANET routing protocols. Our design attempts to target a general-purpose routing algorithm, which seamlessly integrates application layer key-lookup and network layer routing.

We have constructed a mobile model in ns-2 [17] and are implementing MKZR protocols and simulating the system in this environment. We will conduct the evaluation of the performance especially in packet delivery ratio (Data packets ratio) and routing overhead. In the future, our system attempts to supply an effective integrated routing model and protocols for MANETs running a variety of $\mathrm{P} 2 \mathrm{P}$ applications

\section{References}

1. Y.C. Hu, S. Das, and H. Pucha: Exploiting the Synergy between Peer-to-Peer and Mobile Ad Hoc Networks. Proc. HotOS-IX, 9th Workshop on Hot Topics in Operating Systems, Hawaii, May 2003, pp. 18-23.

2. The Gnutella Protocol Specification V0.4 (Document Revision1.2). http://www9.limewire.com/developer/gnutella_protocol_0.4.pdf.

3. B. Zhao, J. Kubiatowicz and A. Joseph: Tapestry: An Infrastructure for Faulttolerant Wide-area Location and Routing. University of California Berkeley Technical Report, 2001.

4. A. Rowstron and P. Druschel. Pastry: Scalable, decentralized object location and routing for large-scale peer-to-peer systems. Proc. IFIP/ACM Int. Conf. on Distributed Systems Platforms (Middleware), Heideberg, 2001, pp. 329-350.

5. I. Stoica, R. Morris, D. Karger, F. Kaashoek and H. Balakrishann: Chord: A Scalable Peer-to-Peer Lookup Service for Internet Applications. Proc. ACM SIGCOMM 2001 - Conference on Applications, Technologies, Architectures, and Protocols for Computer Communication, San Diego, California, 2001, pp. 149-160.

6. S. Ratnasamy, P. Francis, M. Handley, R. Karp, and S. Shenker: A Scalable Content-addressable Network. Proc. IEEE/ACM SIGCOMM 2001 - Conference on Applications, Technologies, Architectures, and Protocols for Computer Communication, San Diego, California, 2001, pp. 161-172.

7. S. Ni, Y. Tseng, Y. Chen and J. Sheu: The Broadcast Storm Problem in a Mobile Ad Hoc Network. Proc. 5th Annual ACM/IEEE Int. Conference on Mobile Computing and Networking (MobiCom'99), Seattle, Washington, 1999, pp. 151-162.

8. C. Lindemann and O. Waldhorst: A Distributed Search Service for Peer-to-Peer File Sharing in Mobile Applications. Proc. IEEE Int. Conf. on Peer-to-Peer Computing (P2P), Linkping, Sweden, 2002, pp. 73-81. 
9. G. Ding and B. Bhargava: Peer-to-Peer File-sharing over Mobile Ad Hoc Networks. Proc. Second IEEE Conf. on Pervasive Computing and Communications Workshops (PERCOMW), Orlando, 2004, pp. 104-108.

10. Z. Haas, M. Pearlman and P. Samar: Zone Routing Protocol - IETF Internet Draft. draft-ietf-manet-zrp-04.txt, Jan. 2001.

11. C.E. Perkins, E.M. Belding-Royer, and S. Das: Ad Hoc On-Demand Distance Vector (AODV) Routing. http://moment.cs.ucsb.edu/pub/rfc3561.txt, IETF RFC 3561, 2003

12. FIPS PUB 180-1, Secure Hash Standard. Federal Information Processing Standard Publication 180-1, National Institute of Standards and Technology, US Department of Commerce, Apr. 1995.

13. M. Papadopouli and H. Schulzrinne: Effects of power conservation, wireless coverage and cooperation on data dissemination among mobile devices. Proc. IEEE/ACM Int. Symp. on Mobile Ad Hoc Networking and Computing (MobiHoc 2001), Long Beach, California, 2001, pp. 117-127.

14. A. Klemm, C. Lindemann and O. Waldhorst: A Special-purpose Peer-to-Peer File Sharing System for Mobile Ad Hoc Networks. Proc. Workshop on Mobile Ad Hoc Networking and Computing, Sophia-Antipolis, France, Mar. 2003.

15. G. Kortuem, J. Schneider, D. Preuitt, T. Thompson, S. Fickas, Z. Segall. When Peer-to-Peer Comes Face-to-Face: Collaborative Peer-to-Peer Computing in Mobile Ad Hoc Networks. Proc. P2P2001, Linkping, Sweden, Aug. 2001.

16. L. Yan, K. Sere and X. Zhou. Towards an Integrated Architecture for Peer-to-Peer and Ad Hoc Overlay Network Applications. Proc. 10th IEEE Int. Workshop on Future Trends of Distributed Computing Systems (FTDCS'04), Suzhou, China, May 2004.

17. The VINT Project, UC Berkeley, LBL and Xerox PARC. The ns-2 manual http://www.isi.edu/nsnam/ns/ns-documentation.html 